
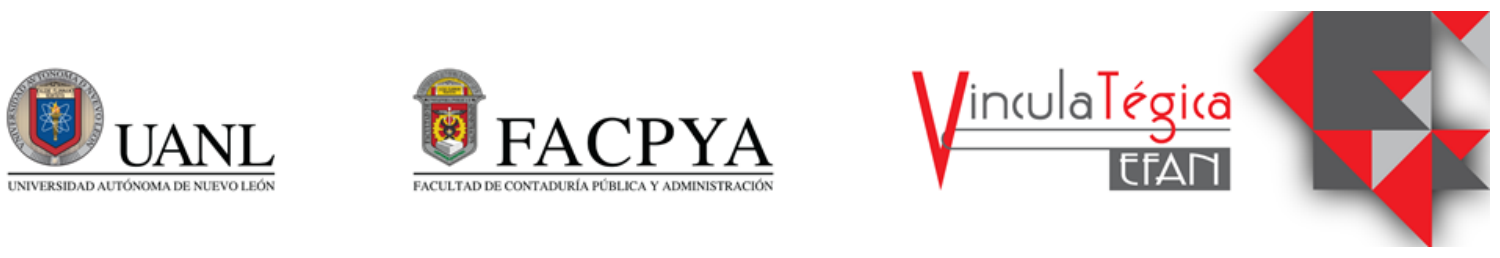

\title{
Diferencias entre hombres y mujeres en el emprendimiento de un micronegocio en México
}

\author{
David Flores Luna \\ ${ }^{1}$ Universidad Autónoma de Nuevo León, davidlri91@gmail.com, Pedro de Alba S/N, Niños Héroes, Ciudad \\ Universitaria, San Nicolás de los Garza, N.L., +52 8183294080.
}

Información del artículo revisado por pares

Fecha de aceptación: junio-2021

Fecha de publicación en línea: diciembre-2021

DOI: https://doi.org/10.29105/vtga7.2-71

\begin{abstract}
Resumen
Las MiPyMES en su totalidad equivalen a más de seis millones de unidades de negocio en México. El gran número de MiPyMES que contribuyen a la generación de empleos y al crecimiento de la economía, ha llevado al gobierno del país a diseñar una serie de programas de apoyo y orientación, así como instrumentos para su conocimiento y análisis, entre los que destacan la Encuesta Nacional de Micronegocios (ENAMIN) ha sido aplicada desde 1992, mediante su levantamiento se ha podido identificar las características de las personas propietarias de estas empresas y las condiciones en las que emprendieron sus negocios. Así el objetivo de este trabajo es realizar un análisis sobre las diferencias entre hombres y mujeres en el emprendimiento de un micronegocio en México, con el fin de entender de forma más detallada y precisa cómo inicia la motivación y la aspiración para que tanto un hombre como una mujer decidan emprender un negocio. Los hombres cuentan con una mayor preparación profesional e integran el grueso de la población empleada en el país, aun cuando las mujeres conforman la mayoría de la población del país, y a pesar de que su papel en la economía ha incrementado de forma notable. Son las diferencias por las que cada grupo decide emprender las que se deben entender para lograr un mejor análisis del contexto del emprendimiento en el país, con el fin de poder ayudar a cada género según sus necesidades para la supervivencia de los micronegocios.
\end{abstract}

\begin{abstract}
The MSMEs in their totality are equivalent to more than six million business units in Mexico. The large number of MSMEs that generate jobs and growth to economy, has led the country's government to the creation and support of guidance programs, as well as instruments for their knowledge and analysis, among which la Encuesta Nacional de Micronegocios (ENAMIN) has been applied since 1992, through its survey it has been possible to identify the characteristics of the people who own these microbusinesses and the conditions in which they started their businesses. Thus, the objective of this work is to carry out a large analysis on the differences between men and women in the entrepreneurship of a micro-business in Mexico, in order to understand in a more detailed and precise way how motivation and aspiration to start a business begin for both man and woman. Men have greater professional training and make up the majority of the employed population, even though in Mexico women make up the majority of population, and despite the fact that their role in the economy has increased significantly. It is in the differences of why each group start a business that they must understand to achieve a better analysis of the context of entrepreneurship in it, in order to be able to help each gender according to their needs for the survival of the micro-businesses.
\end{abstract}

Keywords: microbusiness, entrepreneur, men, women, gender

JEL: E26, J16, J23, L26, L98 
Palabras clave: micronegocios, emprendimiento, hombres, mujeres, genero.

\section{INTRODUCCIÓN}

Las micro, pequeñas y medianas empresas (MiPyMES) en México de acuerdo con el último censo económico de Instituto Nacional de Geografía y Estadística (INEGI) del año 2019 representan en conjunto $99.8 \%$ de todas las empresas del país generan cerca del $70 \%$ de empleos y contribuyen con más del $45 \%$ del Producto Interno Bruto (PIB). Las MIPYMES en su totalidad equivalen a más de seis millones de unidades de negocio. El gran número de MiPyMES que contribuyen a la generación de empleos y al crecimiento de la economía, ha llevado al gobierno del país a diseñar una serie de programas de apoyo y orientación, así como instrumentos para su conocimiento y análisis, entre los que destacan la Encuesta Nacional de Micronegocios (ENAMIN) elaborada conjuntamente por la Secretaría del Trabajo y Prevención Social (STPS) y el INEGI. La ENAMIN ha sido aplicada desde 1992, mediante su levantamiento se ha podido identificar las características de las personas propietarias de estas empresas y las condiciones en las que emprendieron sus negocios.

Las MIPYMES constituyen la columna vertebral de la economía nacional debido a los acuerdos comerciales y apoyos que reciben los empresarios para sus proyectos por parte del gobierno. Sin embargo, en lo que se refiere a los micronegocios familiares, se puede argumentar que constituyen un sector de la economía altamente variado, además, sigue existiendo mucha volatilidad en la creación y el crecimiento de estos negocios ya que, según un estudio de la Secretaría de Economía del gobierno federal tienen un promedio de vida de dos años, por diferentes motivos como la falta de planeación de negocios, insuficiencia de capital, inadecuado uso del financiamiento, vulnerabilidad a la incertidumbre, entre ellos. Por el contrario, un gran número de estudios muestran que la tasa de mortalidad de empresas disminuye a medida que aumenta la edad del negocio; y también señala que la excesiva carga financiera, cambios en la política industrial, falta de capacidad para acceder a nuevos negocios, carecían de habilidades gerenciales, entre otros pueden afectar a los empresas. (Navarrete, 2011).

Por lo tanto, se reconoce la importancia de la generación y la participación de las MIPYMES en el país. Así el objetivo de este trabajo es realizar un análisis sobre las diferencias entre hombres y mujeres en el emprendimiento de un micronegocio en México, con el fin de entender de forma más detallada y precisa cómo inicia la motivación y la aspiración para que tanto un hombre como una mujer decidan emprender un negocio. En la encuesta analizada (la ENAMIN, 2012) se podrán observar los diferentes obstáculos que tienen que sobre llevar tanto hombres como mujeres en el emprendimiento de negocios puesto que como menciona Bonilla (2013) estas últimas enfrentan condiciones más difíciles para desarrollar y consolidar un negocio, lo cual las induce a restringir el tamaño de sus empresas y mantener bajas expectativas de crecimiento, además de que tienen mayor temor al fracaso en todas las actividades económicas y niveles de desarrollo. Por lo tanto, se entiende que en la ENAMIN encontraremos claras diferencias entre hombres y mujeres, así como las circunstancias que influyen en ambos para el emprendimiento de un negocio.

La pregunta de investigación: de acuerdo con la literatura revisada y los aspectos analizados ¿Qué relación existe respecto a la diferencia entre hombres y mujeres en el emprendimiento en México y el nivel mortalidad de las MIPYMES?

Esto ayudará a entender el panorama de los micronegocios en México y ayudar a los tomadores de decisiones a considerar cada punto clave tanto para hombres como para mujeres en el emprendimiento de micronegocios en el país.

Esta investigación tiene como objetivo poner en claro el contexto que gira en torno al emprendimiento en México el conocimiento necesario para su desarrollo, puesto que el 
emprendimiento de negocios es uno de los motores para el crecimiento de la economía de un país. Con esta estructura se pretende desarrollar una investigación lo suficientemente amplia y clara para que más personas puedan comprender de mejor manera la importancia del fenómeno que esta investigación pretende analizar.

\section{MARCO TEÓRICO}

En el presente punto se analizarán los antecedentes históricos y teóricos generales del emprendimiento, el origen y la evolución del tema, así como los tipos de emprendimiento que existen. De igual modo se presentara con detalle el contexto general de los micronegocios en México y por último, se puntualizarán los enfoques teóricos del presente trabajo de investigación.

\subsection{Origen y evolución del emprendimiento}

El estudio del fenómeno de la actividad emprendedora ha sido abordado diferentes perspectivas. Los estudios sobre este fenómeno resaltan que todavía no se ha llegado a un conceso generalizada sobre del término. Autores como Bull y Willard (1993) y Toro y Ortegón (1999) refieren que en más de 200 años de estudios sobre el emprendimiento se ha provisto de una gran variedad de definiciones sobre el concepto; sin embargo, ninguna teoría que se haya desarrollado previamente puede explicar o predecir con exactitud cuándo un emprendedor puede ser definido como tal. Esta confusión sobre la búsqueda de una definición exacta ha impedido que se desarrolle una teoría más precisa sobre el emprendimiento. Por lo tanto, para entender con detalle este tema iniciaremos con el análisis del origen del término "emprendedor" y de su evolución.

Etimológicamente, los términos "emprendedores", "empresa" y el verbo "emprender" provienen del francés entrepreneur, entrepise, y entreprende respectivamente. A su vez, estos términos proceden de la misma raíz del latín vulgar (in, en, y prendere) cuyo significado es coger, atrapar y tomar. (Azqueta, 2017).

La primera vez que se documentó su uso en castellano del verbo emprender se tiene registros de escritos aragoneses de los años 1030 y 1095 con el sentido de "tomo". En el siglo XIII, Gonzalo de Berceo utiliza el concepto en dos pasajes con distintos significados. En el primer caso, como engendrar: "la cepa es buena, emprendió buen sarmiento" En el siglo XVI se da el nombre emprendedor a los que dirigen expediciones militares, capitanes de fortuna que reclutan tropas mercenarias para ponerse al servicio de príncipes, ligas o ciudades. Se resalta la característica propia del emprendedor que aborda una conquista, valiente, con iniciativa y arrojo. Se propicia que, por extensión, el nombre se comienza a aplicar para referir a todo tipo de aventureros. (Azqueta, 2017).

Durante los siglos XVI y XVII el término entrepreneur viene a significar pionero y se refiere a los aventureros que viajan al Nuevo Mundo buscando una nueva oportunidad sin tener en cuenta lo que les espera en esas tierras desconocidas. La primera vez el término fue identificado con actividades económicas ocurrió en el año 1724 en el Dictionnaire universel du commerce de Savary des Brulons. Los hermanos Savary preparan, para su uso personal, una lista alfabética de palabras relacionadas con el comercio y la industria y explican quién es el entrepreneur: "aquel que emprende una obra. Se dice: un empresario de manufactura, un empresario de la construcción; por decir un fabricante, un maestro". En España se refiere a la persona que recibe por encargo real, la producción de un bien o a la que se le confían contratos reales. Esta iniciativa permite que el término se divulgue y facilita que se enriquezca a lo largo del tiempo con aportaciones y acepciones diversas. El término no se generaliza en obras de economía hasta la segunda mitad del siglo XVIII. (Azqueta, 2017).

De acuerdo con Cuervo (1987), se destacan tres acepciones del término emprender en español según el momento histórico y las circunstancias en que han sido 
empleados, que van desde encender el fuego, hasta acometer una acción con arrojo o riesgo:

1) Acometer alguna cosa resuelta $y$ decididamente, con ánimo de ponerla por obra o ejecutarla, por ejemplo: "Puesto que todo sucediese al revés de lo que imagino, la gloria de haber emprendido esta hazaña no la podrá oscurecer malicia alguna (Cervantes, el Quijote)".

2) Un segundo uso es el de "arrojarse" sobre alguna plaza u objetivo militar para atacarlo. También se usa para arrojarse con presteza sobre personas con intención de atacar o arremeter. En algunas ocasiones, el verbo significa entrar en una tierra o introducirse en el sitio atacado.

3) El tercer uso se refiere a "encender el fuego".

El primero en introducir el vocablo entrepreneur en el discurso económico fue Richard Cantillon (1680-1734). Su obra Ensayo sobre la naturaleza del comercio en general (1730) ostenta el mérito de ser el primer escrito de la llamada economía política. En su trabajo, Cantillon reflexiona acerca del entrepreneur, define su perfil y delimita las características del modelo económico que le asiste y que se puede considerar próximo al capitalismo en sus primeras etapas de desarrollo. El principal rasgo que caracteriza al entrepreneur es su capacidad para asumir riesgos en condiciones de incertidumbre. A partir del siglo XVIII la noción de emprendedor corre paralela a la teoría económica y recibe una considerable influencia de la ciencia sociológica en alguna de sus etapas.

\subsection{Concepto de emprendimiento}

El fenómeno de emprendimiento puede definirse, dentro de las múltiples acepciones que existen del mismo, como el desarrollo de un proyecto que persigue un determinado fin económico, político o social, entre otros, y que posee ciertas características, principalmente que tiene una cuota de incertidumbre y de innovación (Formichella, 2004).

Nassir y Reinaldo (Sapag, 1991) definen a un proyecto de la siguiente manera: "un proyecto no es más ni menos que la búsqueda de una solución inteligente al planteamiento de un problema tendiente a resolver, entre tantas, una necesidad humana". Por lo tanto, se puede interpretar que la acción de emprender, al desarrollar un negocio, se busca cubrir las necesidades básicas de cualquier persona como son la alimentación, la salud, la educación, la vivienda, la religión, la política, la cultura, entre otras.

El emprendimiento significa realizar acciones humanas creativas para construir algo de valor a partir de muy pocos recursos. Es la búsqueda insistente de la oportunidad independientemente de los recursos disponibles o de su carencia. Requiere una visión, así como la pasión y el compromiso para guiar a otros en la persecución de dicha visión. También requiere la disposición de tomar riesgos calculados (Timmons \& Zacharakis, 2004).

Según Peter F Drucker (1985) el emprendimiento consiste en maximizar las oportunidades, es decir, la efectividad y no la eficiencia es la esencia del trabajo. La actividad emprendedora es la gestión del cambio radical y discontinuo, o renovación estratégica, sin importar si esta renovación ocurre dentro o fuera de organizaciones existentes si da lugar, o no, a la creación de una nueva identidad de negocio (Kundel, 1991).

Por lo tanto, el emprendimiento se puede definir como ser capaz de crear algo nuevo o de dar un uso diferente a algo ya existente en un ambiente de incertidumbre y, de esta manera, generar un impacto en vida propia y en la comunidad en la que se habita, puesto que se logra desarrollar y alcanzar una visión que logre cubrir todas las necesidades del emprendedor y su entorno.

\subsection{Tipos de emprendimiento}

Las actividades de emprendimiento se expresan de diferentes maneras. Para entender mejor el tema del emprendimiento debemos 
conocer los distintos tipos que existen a través del valor de emprender, considerando que existen diferentes circunstancias para ello, como son la actividad y la capacidad monetaria para invertir. A continuación, se presentarán los diferentes tipos de emprendimiento según Almodóvar (2018).

1) Actividad emprendedora por oportunidad y por necesidad

La actividad emprendedora por oportunidad es aquella que se inicia para explotar alguna oportunidad de negocio. En cambio, el espíritu emprendedor por necesidad aparece cuando no existen alternativas de empleo o estas son insatisfactorias. Ambos se caracterizan por estar en función de la motivación del individuo para emprender.

De acuerdo con los estudios realizados, el emprendimiento se extiende con mayor auge en países con economías más frágiles por la necesidad de su población de buscar un mejor ingreso, mientras tanto en economías con mayor por poder adquisitivo el emprendimiento se realiza fundamentalmente porque se concibe como el momento adecuado, es decir porque existen las condiciones adecuadas para tomar esa decisión. Al respecto, es de suma importancia entender a detalle los motivos que llevan a una persona a emprender, puesto que, los resultados pueden variar dependiendo del contexto que hubo en cada emprendimiento. Algunos autores describen de forma positiva los emprendedores por oportunidad ya que estos resultan favorables para el desarrollo económico mediante la innovación, el suministro de insumos, el aumento del empleo y la productividad con las que están vinculados. En contraste el emprendimiento por necesidad no muestra un gran impacto de desarrollo económico de largo plazo, puesto que estos emprendedores permanecen, en su mayoría, como micronegocios que solamente se convierten en fuentes de ingreso básico para la supervivencia de sus dueños y no deja algún tipo de experiencia para el desarrollo económico, aunque algunos autores critican este tipo de análisis, ya que estipulan que estos emprendimientos pueden ser hallados tanto en países desarrollados como en países en vías de desarrollo, así como en un mismo emprendedor (Almodóvar, 2018).

2) Emprendimiento innovador

El emprendimiento que surge a partir de ideas novedosas a partir de un conocimiento ya existente para mejorarlo se considera un elemento que estimula el desarrollo económico. A nivel particular, si el incentivo implícito para el emprendimiento es la innovación se esperaría un resultado sobresaliente para los comercios. Asimismo en este tipo de emprendimiento es posible aproximar el concepto de iniciativas innovadoras al de iniciativas por oportunidad, y de igual modo este emprendimiento esta mayormente relacionado con países desarrollados que con países subdesarrollados.

En la investigación de Schumpeter (1934) en la que aporta sus ideas sobre el desarrollo económico, el emprendedor/a provoca el proceso de destrucción creativa, ya que, con base a la introducción de innovaciones, como nuevos productos y servicios, nuevas maneras de administración, materias primas, entre otras, se originan cambios con resultados más satisfactorios y se descontinúan las producciones antiguas.

En el emprendimiento innovador podemos toparnos de igual modo con el emprendimiento imitador, un patrón de crecimiento económico el cual no requiere una inversión significativa para investigación ni en tecnología comúnmente encontrada más en países en vías de desarrollo.

3) Clasificación de la actividad emprendedora desde la perspectiva institucionalista

Baumol (1990) expresa que el número de personas dedicadas al emprendimiento es relativamente continua, pero la manera en que ellos se distribuyen depende ampliamente de los gobiernos. Así, podrán surgir tres tipos de emprendimiento; el productivo, el improductivo y el destructivo. Las maneras productivas de emprendimiento tienen el objetivo de crear 
riqueza, las improductivas, reparten la riqueza, aunque no las generan y las destructivas se encarga de destruir la riqueza y de no generala Las instituciones de los países son de suma importancia para el emprendimiento, puesto que, dependiendo de sus políticas públicas pueden afectar directa o indirectamente a los emprendedores.

El círculo virtuoso de emprendimiento y cambio institucional ayuda a despegar a ciertas economías estancadas y este puede surgir de dos formas I) con base en la implementación de enmiendas a las políticas productivas que generan oportunidades a favor de los emprendedores productivos y II) con base a mejoras tecnológicas iniciadas por emprendedores económicos que generan circunstancias adecuadas para los emprendedores políticos productivos. De cualquier forma, se da origen a un proceso en la que el cambio conlleva ocasiones ideales para el otro, y la interacción con este cambio definirá el éxito para la sociedad (Henreckson, 2011) (Henreckson, 2011)

4) Actividad emprendedora local y sistemática

A grandes rasgos Sautet (2013) detalla que la cultura del emprendimiento local está caracterizada por la inexistencia de economías de escala, división de trabajo, acumulación de riqueza y vastas transacciones, por otro lado la actividad emprendedora sistemática la cual se define como la socialmente productiva, que genera economías de escala, involucra la división de trabajo, demanda acumulación de riquezas y la existencia de vastas transacciones. Contrario al emprendimiento local, la sistemática explota grandes redes impersonales. La diferencia más notable entre ambas iniciativas empresariales consiste en las oportunidades disponibles del mercado, es decir, no se refiere al número de oportunidad que se encuentran en el mercado, si no, al éxito que alcanzan dichas oportunidades.

El emprendimiento local se localiza en varios países con economías frágiles. En primer lugar, sus bases se localizan en el sector informal, y esto genera una limitación para la iniciativa empresarial concentrándose únicamente a nivel local. En segundo lugar, se identifica por solo aprovechar las oportunidades locales, y en tercer lugar se caracteriza por estructuras de organización sencillas. Difícilmente llega a extenderse más allá de ámbitos geográficos, debido a que se sostienes de las redes familiares (familiares y amistades).

Mientras el emprendimiento sistemático existen conexiones, también sistemáticas, que posibilitan intercambios comerciales entre las personas que no tienen un vínculo cercano y se encuentran en diferentes espacios geográficos a veces en espacios muy lejanos y en una amplia redes de negocio.

5) Actividad emprendedora formal e informal

Otra manera de catalogar la iniciativa empresarial se da en función del grado de formalidad de las actividades que lleva a cabo. Así, se puede definir al emprendedor no formal, como la persona que participa en el desarrollo de un nuevo negocio dedicado a la comercialización de bienes y servicios, pero, que no está aún registrado con las instituciones gubernamentales. Mientras que el emprendimiento formal se caracteriza como el desarrollo de nuevos comercios que están registrados adecuadamente ante una institución gubernamental.

El emprendimiento informal es de vital importancia debido a tres principales razones: 1) es un fenómeno que representa el 30\% de la economía mundial, 2) afecta a todo tipo de economía y 3) es altamente prevalente en ciertos países. Que un negocio sea informal ni implica necesariamente que ofrezca bienes y servicios ilegales, es más, pueden comerciar con bienes y servicios lícitos, pero el hecho de que no esté registrado de manera oficial ante el gobierno podría limitar seriamente su crecimiento (Thai, 2014).

\subsection{Los micronegocios en México}

Los micronegocios son unidades económicas que emplean a menos de 10 personas En 
México según el censo económico del 2019 realizado por el INEGI existen aproximadamente 4,555,263 micronegocios distribuidos en todo el territorio nacional, los cuales ocupa al $37.2 \%$ de toda la población económicamente activa del país, superando los 10 millones de personas. De igual modo su participación en el PIB es de apenas el 14.6\%.

Según datos de una encuesta realizada en línea por la Asociación de Emprendedores de México (ASEM, 2020), en el perfil de los emprendedores del país destaca que el 39\% de los emprendimientos son de mujeres contra el $61 \%$ de hombres De igual modo casi dos terceras partes de los emprendedores oscila en una edad de 26 a 45 años, y alrededor del $90 \%$ tiene algún estudio universitario. Más del $80 \%$ de los negocios facturan menos de dos millones de pesos al año. Y el 38\% de las empresas fue fundado por una sola persona, mientras que el $40 \%$ fue fundado por dos personas y el resto por 3 o más personas.

En México los principales motivos para emprender una empresa son los deseos de crecimiento profesional y personal. Este hecho puede relacionarse con la idea de que un emprendedor es una persona con deseos de obtener mayor crecimiento y superación, a diferencia del resto de las personas que no realiza algún tipo de emprendimiento. En segundo lugar, con el $31 \%$ tenemos el motivo solucionar problema del ambiente o del mercado, aquí podemos encontrar la innovación de los emprendedores los cuales deciden llevar a cabo un negocio con alguna novedad que puede cambiar el juego del mercado al que desean entrar y con el que podrían tener un diferenciador que los ayude a sobrevivir y más adelante a resaltar. En tercer lugar, encontramos la oportunidad rentable, es decir un tiempo y una circunstancia favorables para emprender un negocio. En cuarto lugar se ubica el motivo incrementar el nivel de ingreso, que puede ir muy relacionado al crecimiento profesional y personal. Y con el 20\% tenemos el motivo desarrollar un producto o servicio, que también puede estar vinculado con la innovación, puesto que, además de resolver un problema, estos emprendedores están interesados con innovar con algún producto que ayude a revolucionar el mercado.

De acuerdo con el INEGI (2019) las microempresas son el grupo de empresas, separadas por tamaño, que menos invierte en la capacitación de su personal (apenas el 2.4\%). El $48.1 \%$ de las personas laborando para micronegocios cuenta solo con educación básica, mientras que el $40 \%$ es mayor de 41 años. Dentro del rubro de rotación de personal, estos negocios tienen los porcentajes más bajos (apenas el 14.6\%) obteniendo mayor fidelidad de sus empleados que las empresas grandes. Esto último también podría deberse a los altos costos y la poca preparación para reclutar personal adecuado para el negocio.

Del total de los micronegocios en México únicamente el $11.4 \%$ logró obtener algún tipo de financiamiento por parte de una institución financiera. El $20 \%$ de estos negocios cuenta con algún equipo de cómputo, solo $17.4 \%$ tiene acceso a internet, y un porcentaje mucho más bajo, solo el $2.1 \%$ tuvo ventas por internet. De acuerdo a cálculos del censo económico en promedio los micronegocios generaron al año 2019, 278 mil pesos en activos fijos anualmente (INEGI, 2019).

En ese mismo año más del $62 \%$ de los establecimientos se encontraban en un estatus informal, es decir no estaba registrado ante ninguna institución oficial. También, estos establecimientos se caracterizan por no tener a más de cinco personas empleadas, no pagar seguridad social ni prestaciones, no llevar un sistema contable, y no pagar por asesoría comercial, entre otros. Estas características pueden representar limitantes para su crecimiento y supervivencia, lo cual provoca que también sean el grupo de empresas que menor remuneración otorga a sus empleados con solo 62 mil pesos anuales contra los 186 mil que ofrecen en promedio las empresas más grandes (INEGI, 2019).

Este problema se acentúa en el proceso para realizar trámites y servicios federales según un estudio realizado por el Instituto Mexicano de la Competitividad (IMCO, 2019) los gastos por dichos tramites representan el $2.7 \%$ del PIB del país, por ejemplo el costo de regulaciones federales en 
el año 2018 fue de 635 mil millones de pesos. De igual modo de acuerdo con el análisis de IMCO un trámite federal y/o estatal tarda en promedio una hora y media, mientras los trámites a nivel municipal hasta ocho horas; tomando en cuenta que los micronegocios deben tratar primordialmente con este último nivel gubernamental, esta situación dificulta su cambio a un estatus formal.

Por otra parte los micronegocios al igual que el resto de los negocios detallaron que la inseguridad pública al año 2019 era el principal problema que enfrentaban para llevar a cabo su actividad, al menos así lo indico más del $40 \%$ de los dueños entrevistados; le siguen los altos gastos de servicios, altos impuestos, exceso de trámites gubernamentales, costos de materias primas, insumos o mercancías, y por último la competencia desleal (INEGI, 219).

\subsubsection{Características de las mujeres al frente de un micronegocio}

De acuerdo al análisis realizado por Instituto Nacional de las Mujeres (INMUJERES) con base en la Encuesta nacional de Ocupación y Empleo (ENOE) del 2019, las mujeres empleadoras en México pertenecen en su mayoría al servicio terciario, con un $83.7 \%$, $13 \%$ al secundario y $3.4 \%$ al primario. De este mismo número el $54.7 \%$ se encuentra en zonas más urbanizadas (INEGI considera como zonas más urbanizadas aquellas que cuentan con más de 100 mil habitantes), y el resto en las menos urbanizadas. El 91.9\% de las mujeres están al frente de micronegocios y $8.4 \%$ son empleadoras de un negocio pequeño.

De igual manera el $49.2 \%$ de las mujeres obtiene ingresos de hasta dos salarios mínimos contra el $34.6 \%$ de los hombres, y tan solo el $7.1 \%$ de las mujeres percibe ingresos mayores a los cinco salarios mínimos, contra el $14.4 \%$ de los hombres. El $73 \%$ de las mujeres emprendedoras tienen una edad que oscila entre los 30 y 59 años. Respecto a la escolaridad el $42.5 \%$ de las mujeres tiene educación medio superior o superior contra el $38.6 \%$ de los hombres. El estudio afirma que en un $64.3 \%$ las mujeres están casadas o viven en unión libre, mientras que en un $20 \%$ están separadas, divorciadas o viudas y $14.9 \%$ son solteras. Por último, el $33.4 \%$ está conformado por jefas de hogar contra el $82.6 \%$ integrado por los jefe de hogar hombres (INMUJERES, 2019)

\section{MÉTODO}

Este apartado se conforma por la metodología y el diseño de la investigación implementada para poder obtener los resultados acerca de las hipótesis establecidas y así posteriormente llegar al análisis de las diferencias entre hombres y mujeres en el emprendimiento de micronegocios en México, así como al orden de la recurrencia de la diferencia de los distintos motivos entre ambos grupos para emprender. Para alcanzar nuestro objetivo principal, se recurre la Encuesta Nacional de Micronegocios (ENAMIN) aplicada por el INEGI en el 2012.

\subsection{Metodología de la investigación}

La investigación que desarrollaremos será de tipo cuantitativa y correlacional. Con ésta investigación buscamos conocer el grado de las variaciones de los factores, en relación con otros factores para determinar la recurrencia entre mujeres y hombres de los motivos para emprender micronegocios en México y sus diferencias. La investigación es cuantitativa, lo cual nos permitirá conocer la realidad de una manera imparcial debido a que se recogen y analizan los datos a través de los conceptos $\mathrm{y}$ las variables estudiadas.

\subsection{Diseño de la investigación}

En este estudio se elaboró un diseño no experimental, ya que se limita a la observación de la conducta de las variables que se desean explicar sin sufrir manipulación, es decir, tal y como sucedieron en su contexto natural. La investigación tiene una cohorte transversal o transeccional pues analiza la información estadística para describir y explicar las relaciones existentes entre las variables en un punto o momento de tiempo a estudiar (Hernández et al., 2016). 


\subsection{Fuentes de la información y variables}

La presente investigación analiza la información nacional recolectada por el INEGI a través de la ENAMIN para el año 2012, en los sectores económicos de manufactura, comercio, construcción y servicios de todo el país.

La encuesta diseñada por el INEGI es de tipo estructurada y fue creada para obtener información de las microempresas en las que laboran de una a seis personas incluyendo al dueño y hasta 16 si se trata de empresas del sector manufacturero. La encuesta se aplica utilizando la técnica de encuesta estructurada, administrada y personal a nivel nacional.

La ENAMIN se ha aplicado en ocho ocasiones en los siguientes años: 1992, 1994, 1996, 1998, 2002, 2008, 2010 y 2012. Los cuestionarios para los ocho años son ligeramente diferentes en los distintos años de su aplicación. También varían el número de empresas que el INEGI encuestó en cada levantamiento de información. Estos cuestionarios tienen variaciones que van de las 71 a las 100 preguntas según el año de su aplicación. Como se ha mencionado, la presente investigación estudia a nivel nacional los resultados correspondientes al año 2012. Asimismo esta investigación sólo se trabaja con las secciones y las preguntas de la ENAMIN que resultaron del eje temático de financiamiento; en el Anexo 1 se muestra dicha sección.

\section{RESULTADOS}

En este punto se dan a conocer los resultados de los instrumentos definidos del punto previo. Asimismo, se muestran los estadísticos descriptivos de la base de datos. Por último, en la discusión de los resultados se presenta un análisis de investigaciones elaboradas sobre el tema estudiado.

\subsection{Presentación de los resultados obtenidos}

En las siguientes secciones se mostrarán los datos recopilados de la ENAMIN. Primero, se presenta una tabla con los estadísticos de las personas entrevistadas, a través de la cual se pretende analizar el número de estas personas, saber cuántos de estos datos fueron válidos para el análisis, además de presentar la media, la desviación estándar, el mínimo y el máximo de cada pregunta.

Posteriormente, encontraremos una tabla con las primeras tres preguntas de los datos recopilados los cuales en conjunto recolectan las características de las personas entrevistadas, como son el género, la edad y el estado civil. Inmediatamente, mostraremos las Tablas 2, 3 y 4 en las que se detallan características de los negocios. Por último, tenemos un cuadro comparativo de las principales diferencias en los motivos de hombres y de mujeres en el momento de elegir el emprendimiento.

En la Tabla 1 como se puede observar en los datos recopilados respecto al género prácticamente, del número de encuestados, la mitad es del sexo masculino y la otra mitad del sexo femenino. Esto puede ser de mucha ayuda para lograr un análisis más certero sobre la diferencia entre hombres y mujeres puesto que, tener un número muy similar de encuestados de ambos grupos, permite tener una mejor apreciación del caso. En el mismo sentido, es notorio que las mujeres encuestadas representan $0.4 \%$ superior al de los hombres, con lo que se puede señalar que el número de mujeres empieza a superar al de los hombres en los emprendimiento en el país. Esto último va a la par del porcentaje de población de hombres y mujeres en México, según el último censo del INEGI en el año 2020 el $51.2 \%$ de la población está conformada por mujeres contra el $48.8 \%$ que procede de la población masculina.

Respecto a la sección que identifica a las personas entrevistadas según su edad en esta tabla se decidió distribuir a estas personas en nueve grupos con rangos de nueve años cada uno, por ejemplo, de los 12 a los 20 o de los 30 a 38: solamente el último grupo contempla un rango de 15 años, debido a que tiene menos personas encuestadas y con el fin de simplificar la Tabla.

En este apartado podemos observar que los grupos más numerosos abarcan las 
edades de 30 a 38, 39 a 47 y de 48 a 56, y representan dos terceras partes de la población encuestada. Esto nos puede ayudar a inferir que la mayoría de las personas que deciden emprender algún negocio oscila entre estas edades. Asimismo, lo que las caracteriza es que por lo general tienen familias, es decir personas que dependen económicamente de ellas, como niños y adultos mayores por lo tanto, buscan algún ingreso que ayude a solventar los gastos de sus familias lo cual podría ser su mayor motivo para emprender un negocio.

Tabla 1. Características de las personas entrevistadas

\begin{tabular}{|c|c|c|}
\hline \multicolumn{3}{|c|}{ Características de las personas entrevistadas } \\
\hline Género & Frecuencia & Porcentaje \\
\hline Hombre & 12,392 & $49.6 \%$ \\
\hline Mujer & 12,583 & $50.4 \%$ \\
\hline Total & 24,975 & $100 \%$ \\
\hline $\begin{array}{l}\text { Edad de las } \\
\text { personas. }\end{array}$ & Frecuencia & Porcentaje \\
\hline 12 a 20 & 382 & $1.6 \%$ \\
\hline 21 a 29 & 2,691 & $10.8 \%$ \\
\hline 30 a 38 & 5,038 & $20.1 \%$ \\
\hline 39 a 47 & 6,172 & $24.7 \%$ \\
\hline $\begin{array}{l}48 \text { a } 56 \\
57 \text { a } 65 \\
66 \text { a } 74 \\
75 \text { a } 83 \\
84 \text { a } 98 \\
\text { Total }\end{array}$ & $\begin{array}{l}5,321 \\
3,371 \\
1,450 \\
453 \\
97 \\
24,975\end{array}$ & $\begin{array}{l}21.5 \% \\
13.5 \% \\
5.8 \% \\
1.8 \% \\
0.3 \% \\
100 \%\end{array}$ \\
\hline $\begin{array}{l}\text { Estado Civil } \\
\text { Vive con su } \\
\text { pareja en unión } \\
\text { libre }\end{array}$ & 3,634 & $14.6 \%$ \\
\hline $\begin{array}{l}\text { Está separado } \\
\text { (a) }\end{array}$ & 1,436 & $5.7 \%$ \\
\hline $\begin{array}{l}\text { Está divorciado } \\
\text { (a) }\end{array}$ & 736 & $2.9 \%$ \\
\hline Está viudo (a) & 1,458 & $5.8 \%$ \\
\hline $\begin{array}{l}\text { Está casado (a) } \\
\text { Está soltero (a) }\end{array}$ & 14,077 & $56.4 \%$ \\
\hline Perdidos & 3,633 & $14.5 \%$ \\
\hline & $\begin{array}{l}1 \\
24,975\end{array}$ & $\begin{array}{l}0 \\
100\end{array}$ \\
\hline
\end{tabular}

Fuente: elaboración propia.
En la última parte de la tabla anterior se muestra el estado civil de los emprendedores encuestados, es decir si se encuentran casados o en unión libre, si son viudos, divorciados, etc. Como podemos observar en la Tabla 1 el grupo más grande en gran proporción es el de casados seguido por el grupo de quienes viven en unión libre. Por lo tanto se puede inferir que las personas que se encuentran en estos grupos, con mucha probabilidad tienen una familia con más de dos integrantes de manera que sus gastos son mayores a los de aquellos que viven solas. Esto tiene relación con las personas que se encuentran entre los 30 y 45 años lo cual genera como motivo mayor para emprender un negocio el poder solventar los gastos del hogar.

En la encuesta se pidió a las personas entrevistadas que señalaran el número de empleados que laboraban en sus micronegocios. Al respecto en la tabla 2 vemos que aproximadamente el 52\% de los datos fue válido, y de estos podemos constatar que el grupo más grande está conformado por negocios con solo dos personas. En consecuencia estos negocios probablemente no tienen altos ingresos debido a que su operación requiere de pocos elementos, además, esto puede suponer que son negocios especializados que, por lo general, solo requieren de una persona con habilidades y conocimientos específicos, por ejemplo, un taller mecánico o un consultorio médico.

Tabla 2. Datos generales del encuestado. ¿Aproximadamente cuántas personas, incluyendo al dueño, laboraban donde usted trabajaba?

\begin{tabular}{ccc}
\hline $\begin{array}{c}\text { Número de } \\
\text { trabajadores }\end{array}$ & Frecuencia & Porcentaje \\
\hline 1 & 1,471 & $5.90 \%$ \\
2 & 5,429 & $21.70 \%$ \\
3 & 1,283 & $5.10 \%$ \\
4 & 571 & $2.30 \%$ \\
5 & 503 & $2 \%$ \\
6 & 469 & $1.90 \%$ \\
7 & 543 & $2.20 \%$ \\
8 & 683 & $2.70 \%$
\end{tabular}




\begin{tabular}{ccc}
9 & 596 & $2.40 \%$ \\
10 & 357 & $1.40 \%$ \\
11 & 902 & $3.60 \%$ \\
99 & 204 & $0.80 \%$ \\
Total & 13,011 & $52.10 \%$ \\
Perdidos & 11,964 & $47.90 \%$ \\
Total & 24,975 & $100 \%$ \\
\hline Fuente: elaboración propia con datos de la ENAMIN \\
(2012).
\end{tabular}

Como podemos reflexionar a partir de la Tabla 3 en su mayoría las personas encuestadas deciden emprender solas, es decir una persona sola emprende un negocio prefiriendo realizar esta actividad por si misma que en equipo, y provocando que muchas de las tareas recaigan en ella. Esto puede influir significativamente en los resultados de las empresas, debido a que una persona, por lo general, posee habilidades, conocimientos y experiencia limitados, lo que podría ser una desventaja si se compara con lo que un grupo de personas, podría en conjunto, poseer.

Tabla 3. Datos generales del encuestado. ¿Este negocio o actividad es de cuantos dueños?

\begin{tabular}{ccc}
\hline & Frecuencia & Porcentaje \\
\hline Varios Dueños & 23880 & $4.4 \%$ \\
Un solo dueño & 1095 & $95.6 \%$ \\
Total & 24,975 & $100 \%$ \\
\hline
\end{tabular}

Fuente: elaboración propia con datos de la ENAMIN (2012).

En la Tabla 4, la última de los estadísticos se refiere la pregunta sobre que la forma de asociación que tienen los involucrados en el negocio. Como se señaló en la Tabla anterior únicamente 1,095 personas contestaron que su emprendimiento era en conjunto, y esta es la razón principal por la que esta última variable tiene el menor número de datos válidos. Por consiguiente, se puede observar que la mayoría de los encuestados señaló que decidió realizar su emprendimiento con algún miembro de su familia; siendo esta una pregunta abierta se puede inferir que el emprendimiento se lleva cabo entre hermanos, primos, papa e hijo, tío y sobrina, etc. Y debido a que este grupo es el más numeroso también se puede inferir que en México las personas tienen mayor grado de confianza con miembros de su familia. En el segundo grupo, con menos de la mitad de personas encuestadas tenemos que el negocio se formó con personas fuera de su familia, es decir, con un amigo o un conocido. Por último, con un porcentaje mucho menor están las asociaciones con dos o más miembros de una familia y con al menos una persona que no forma parte de esa familia.

Tabla 4. Datos generales del encuestado.

La forma de asociación del micronegocio

\begin{tabular}{ccc}
\hline Tipo & Frecuencia & Porcentaje \\
\hline Familiar & 23880 & $2.9 \%$ \\
No Familiar & 1095 & $1.30 \%$ \\
Familiar y no & 35 & $0.10 \%$ \\
familiar & & $95.6 \%$ \\
Total & 1095 & $4.40 \%$ \\
Perdidos & 23880 & $95.6 \%$ \\
Total & 24,975 & $100 \%$ \\
\hline
\end{tabular}

Fuente, elaboración propia con datos de la ENAMIN (2012).

En la Tabla 5 se puede apreciar que los hombres se conforman casi el $30 \%$ de la población encuestada quienes señalaron que el motivo principal por el que iniciaron su emprendimiento fue poder mejorar el ingreso monetario percibido para sus gastos. Por lo tanto, se puede deducir que los negocios son vistos por ellos principalmente como un paso para obtener mejores ingresos $\mathrm{y}$, por ende, una mayor capacidad de compra y con esto, probablemente, una mejor calidad de vida para el emprendedor y las personas que forman parte de su familia.

En segundo lugar, encontramos que los hombres deciden emprender un negocio para poder desarrollar y poner en práctica sus conocimientos, habilidades y experiencias como se había mencionado anteriormente: estos negocios pueden ser un taller mecánico, consultorio médico, bufete de abogados, los cuales también pueden ser desempeñados por mujeres. En el tercer grupo con más respuestas el otro motivo fue: otra razón, aquí cabria referir decisiones personales como una meta que se impuso el emprendedor de poseer su propio negocio y aprovechar una oportunidad para invertir en un negocio, por tener metas 
Tabla 5 Cuadro comparativo entre hombres y mujeres sobre

: ¿Cuál es el motivo principal por el que se inició este negocio o actividad?

\begin{tabular}{|c|c|c|c|c|}
\hline & Hombre & & Mujer & \\
\hline Motivo principal para emprender. & Frecuencia & Porcentaje & Frecuencia & Porcentaje \\
\hline Por tradición familiar o lo heredó & 987 & $8.0 \%$ & 582 & $4.6 \%$ \\
\hline $\begin{array}{l}\text { Para complementar el ingreso } \\
\text { familiar }\end{array}$ & 1198 & $9.7 \%$ & 5226 & $41.5 \%$ \\
\hline Para mejorar el ingreso & 3283 & $26.5 \%$ & 2133 & $17.0 \%$ \\
\hline $\begin{array}{l}\text { Tenía dinero y encontró una buena } \\
\text { oportunidad }\end{array}$ & 739 & $6.0 \%$ & 480 & $3.8 \%$ \\
\hline $\begin{array}{l}\text { Para ejercer su oficio, carrera o } \\
\text { profesión }\end{array}$ & 1999 & $16.1 \%$ & 797 & $6.3 \%$ \\
\hline $\begin{array}{l}\text { Fue la única manera que tuvo para } \\
\text { obtener un ingreso }\end{array}$ & 1507 & $12.2 \%$ & 1260 & $10.0 \%$ \\
\hline $\begin{array}{l}\text { No tenía la experiencia requerida } \\
\text { para un empleo }\end{array}$ & 22 & $.2 \%$ & 22 & $.2 \%$ \\
\hline $\begin{array}{l}\text { No tenía la escolaridad o la } \\
\text { capacitación requerida para un } \\
\text { empleo }\end{array}$ & 69 & $.6 \%$ & 31 & $.2 \%$ \\
\hline $\begin{array}{l}\text { Estaba sobrecapacitado para un } \\
\text { empleo }\end{array}$ & 13 & $.1 \%$ & 1 & $.0 \%$ \\
\hline $\begin{array}{l}\text { Los empleos que encontró estaban } \\
\text { mal pagados }\end{array}$ & 195 & $1.6 \%$ & 33 & $.3 \%$ \\
\hline Requería un horario fléxible & 138 & $1.1 \%$ & 337 & $2.7 \%$ \\
\hline No había oportunidades de empleo & 899 & $7.3 \%$ & 312 & $2.5 \%$ \\
\hline Otra razón & 1343 & $10.8 \%$ & 1369 & $10.9 \%$ \\
\hline Total & 12392 & $100.0 \%$ & 12583 & $100.0 \% \mathrm{x}$ \\
\hline
\end{tabular}

Fuente: elaboración propia con datos de la ENAMIN (2012).

económicas y en el ámbito social, se pueden tomar en cuenta las circunstancias que lo llevaron a tomar esa decisión que, como mencionan algunos autores podrían ser la pérdida de empleo, ser despedido, cambio de residencia, etcétera.

En el caso de las mujeres como se muestra en la Tabla 5 más de la tercera parte menciona que el emprendimiento se debió a que buscaban complementar el ingreso familiar. En otras palabras, se puede inferir que las mujeres cuentan con otro miembro de la familia económicamente activo que tiene un trabajo, pero cuyos ingresos no son suficientes para solventar los gastos del hogar, por eso deciden emprender uno propio. En segundo lugar. Encontramos que fue para mejorar el ingreso, este puede ir de la mano con lo explayado al principio del párrafo, al ser insuficiente el ingreso del miembro de la familia que posee un puesto de trabajo en una empresa o incluso un negocio, la mujer emprende el negocio para obtener un mayor ingreso monetario.

\section{CONCLUSIONES}

En función a responder a la pregunta de investigación de la diferencia en el emprendimiento entre hombres y mujeres en México y analizando el fenómeno que motiva a unos y a otras en el país para iniciar un negocio en el país, se puede concluir que las personas tienen un sin fin de motivos para crear un emprendimiento, pero con el claro objetivo de generar una fuente de ingresos que funcione primordialmente, como principal herramienta para adquirir una mejor calidad y el estilo de vida deseada por el emprendedor o emprendedora.

En el caso de hombres y mujeres notamos 
que la mayor diferencia se aprecia en la Tabla número 5, más del 50\% de las mujeres emprendedoras deciden iniciar un negocio para ayudar a complementar el gasto familiar

O para mejorarlo, por lo tanto se puede inferir que este grupo de mujeres en su mayoría no son, quizás, la cabeza de la familia es decir muchas veces este tipo de negocios no son la fuente principal de ingresos provocando que sus esfuerzos no se orienten en desarrollar las bases necesarias para que sus emprendimientos sobrevivan el paso del tiempo.

En el desarrollo de la investigación fue posible apreciar que los diferentes teóricos explican que uno de los principales motores para el emprendimiento es el desempleo, es decir las personas al no encontrar un empleo formal en alguna de las grandes empresas (con miles de empleados), deciden crear su propia empresa para obtener sus propios ingresos y dejar de pertenecer gracias a sus medios particulares a la población desempleada, por lo tanto cuando un negocio no nace para cubrir la necesidad del desempleo muchas veces las personas limitan sus capacidades y esfuerzos para desarrollar sus emprendimientos, esto puede afectar la supervivencia de los micronegocios que a la vez afecta el rendimiento de la actividad económica del país. Puesto que, este tipo de negocios ayuda a reactivar la economía de un país, ya que permite la circulación de dinero con la compra y venta de bienes y servicios. De igual modo puede llegar a generar empleos, lo cual conforma otra manera de aumentar la actividad económica.

De igual modo, respecto a la composición de las familias en México es decir, en su mayoría las cabezas de familias, (la persona que provee de más ingresos al hogar) son hombres, puesto que como se pudo observar durante el desarrollo de la investigación, ellos cuentan con una mayor preparación profesional e integran el grueso de la población empleada en el país, aun cuando las mujeres conforman la mayoría de la población del país, y a pesar de que su papel en la economía ha incrementado de forma notable. Son estas pequeñas diferencias las que se deben entender para lograr un mejor análisis del contexto del emprendimiento en el país, con el fin de poder ayudar a cada género según sus necesidades para la supervivencia de los micronegocios.

En consecuencia, los resultados obtenidos en este trabajo de investigación se puede apreciar que el objetivo principal de este estudio se consiguió, se logró entender cómo afecta la diferencia entre hombres y mujeres al emprendimiento de micro negocios en México, pero todavía se encuentran algunas limitación en este trabajo, por ejemplo, cuáles serían las estrategias adecuadas para ayudar a cada grupo a la supervivencia de sus micronegocios y como estas pueden ser impulsadas para que a la vez estos ayuden al crecimiento de la economía.

\subsection{Recomendaciones}

El contexto en el que se basó este estudio fue llevado a cabo en el año de 2012 un año en el que el país experimento un crecimiento económico de casi el $4 \%$ según datos del Banco de México, de modo que existía un panorama positivo para la economía y de expansión. Ahora en el año que se escribe esta investigación, en el 2021, han sucedido acontecimientos que han causado graves daños a la economía mundial y nacional provocados por la pandemia del COVID-19. En consecuencia con la pérdida de empleos y de ingresos en los hogares de las familias mexicanas se espera que muchas personas decidan entrar al emprendimiento para lograr mejorar su calidad de vida, pero para ambos casos los motivos principales pueden diferir puesto que aún existen muchos hogares donde los hombres son la cabeza de la familia y la principal fuente de ingresos. Es por esto que se recomienda que el gobierno tome decisiones con base a estudios como el presente para implementar políticas públicas que ayuden a mejorar el rendimiento y fomentar la calidad y la innovación con el fin de ayudar a la supervivencia de las MIPYMES, las cuales serán el motor para la recuperación y posterior crecimiento de la economía nacional. 


\section{REFERENCIAS}

Almodóvar, M, (2018). Tipo de emprendimiento y fase de desarrollo como factores clave para el resultado de la actividad emprendedora. Cuadernos de relaciones laborales, 36(2), 225244.

Alvarado, E., Ortiz, J. y Morales, D. (2018). Factores que influyen en el emprendimiento de un micronegocio familiar en México. Revista de Economía del Rosario, 21 (1), 153-180.

Asociación de Emprendedores de México. (2020). Radiografía del Emprendimiento en México 2020: Parte 1 Datos Generales. Doi: https://drive.google.com/file/d/176S44oaRiPfSM2gnwfRZDh-YX3Yk4jsD/view

Audretsch, D.B. \& Fritsch, M. (1994). The geography of firm birth in Germany. Regional Studies, 28(4), 359-364.

Azqueta A. (2017). El concepto de Emprendedor: Origen, evolución e interpretación. En Simposio Internacional El Desafío de Emprender en la Escuela del Siglo XXI, 21-39: https://idus.us.es/bitstream/handle/11441/74177/El\%20concepto\%20de\%20emprendedo r\%20origen $\% 2 \mathrm{C} \% 20$ evoluci\%C3\%B3n\%20e\%20introducci\%C3\%B3n.pdf?sequence $=1$ \&isAllowed=y

Banco de México (2016). Situación Económica Internacional y sus implicaciones para la Economía de Mexicana, Asociación de Empresarios Mexicanos, [Capítulo Laredo]: https://www.banxico.org.mx/publications-and-press/presentations/\%7B41DAFF86E7A2-FBAD-7C4F-729778268C7F\%7D.pdf

Baumol, W. (1990). Entrepreneurship: Productive, Unproductive and Destructive. Journal of Political Economy, 98(5): 893-921. https://delong.typepad.com/baumol-1990entrepreneurship.pdf

Brenes, L. \& Bermúdez, L. M. (2013). Diferencias de Género en el emprendimiento empresarial $\begin{array}{llll}\text { costarricense. } & \text { Tec } & \text { Empresarial, } & \text { 7(2), }\end{array}$ https://revistas.tec.ac.cr/index.php/tec_empresarial/article/view/1510

Bull, I. \& Willard, G. E. (1993). Towards a Theory of Entrepreneurship, Journal of Business Venturing, 8 , 183-195. https://citeseerx.ist.psu.edu/viewdoc/download?doi=10.1.1.458.709\&rep=rep1\&type=pd $\mathrm{f}$

Cuervo, J. R. (1987). Diccionario de Construcción y Régimen de la Lengua Castellana, Instituto Caro y Cuervo.

Formichella, M. M. \& Massigoge, J. I. (2004) El concepto de emprendimiento y su relación con la educación, el empleo y el desarrollo local, Instituto Nacional de Tecnología Agropecuaria (INTA).

http://municipios.unq.edu.ar/modules/mislibros/archivos/MonografiaVersionFinal.pdf

Gartner, W. (1985). A Conceptual Framework for Describing the Phenomenon of New Venture Creation. The Academy of Management Review, 10(4), 696-706. https://www.jstor.org/stable/258039?origin=crossref\&seq=1

Henreckson, M. \& Sanandaji, T. (2011). The interaction of entrepreneurship and institutions. Journal of Institutional Economics, 7, 47-75.

Hernández, R., Fernández, C., \& Baptista, P. (2016). Métodos de Investigación: General. McGrawHill

Hidalgo, L. (2014). La Cultura del Emprendimiento y su formación. Red de Revistas Científicas de América Latina, el Caribe, España y Portugal, 3(2), 1-8. https://www.redalyc.org/pdf/5523/552357189003.pdf

Instituto Nacional de Estadística y Geografía. (2019) Censos Económicos 2019: Resultados Definitivos. https://www.inegi.org.mx/contenidos/programas/ce/2019/doc/pprd_ce19.pdf Instituto Nacional de Estadística y Geografía. (2019) Censos Económicos 2019: Características de 
los

negocios.

https://www.inegi.org.mx/contenidos/programas/ce/2019/doc/frrdf_ce19.pdf

Instituto Nacional de las Mujeres. (2019). Empleadoras. http://estadistica.inmujeres.gob.mx/formas/tarjetas/Empleadoras.pdf

Keeble, D. \& Walker, S. (1994). New Firms, small firms and dead firms spatial patterns and determinants in the United Kingdom. Journal Regional Studies, 28: 411-427

Kritikos, A. S. (2014). Entrepreneurs and their impact on jobs and economic growth. IZA World of Labor 2014, 8. https://wol.iza.org/uploads/articles/8/pdfs/entrepreneurs-and-their-impacton-jobs-and-economic-growth.pdf? $\mathrm{v}=1$

Kuratko, D. F. (2004), Entrepreneurship Education: Emerging Trends and Challenges for the 21st Century, 2003 Coleman Foundation White Paper Series for the U.S. Association of Small Business \& Entrepreneurship. https://www.unm.edu/ asalazar/Kauffman/Entrep_research/e_ed.pdf

Melián N. A. \& Campos C. V. (2009). Emprendedurismo y Economía Social como Mecanismos de Inserción Sociolaboral en Tiempos de Crisis. Revista de Estudios Cooperativos, 100 (Extraordinario), 43-67. https://revistas.ucm.es/index.php/REVE/article/view/19552

Navarrete E. M. \& Sansores E. G. (2011). El fracaso de las Micro, Pequeñas y Medianas Empresas en Quintana Roo, México: Un análisis multivariante. Revista Internacional $\begin{array}{llll}\text { Administración } & \text { y } & \text { Finanzas, 21-33. }\end{array}$ http://dx.doi.org/10.12804/revistas.urosario.edu.co/economia/a.6816

Organización de las Naciones Unidas para la Educación, la Ciencia y la Cultura. (1998). Educación Superior en el Siglo XXI: Visión y acción. http://sigc.uqroo.mx/Documentos\%20Externos/Educacion\%20Siglo\%20XXI\%20UNES CO.pdf

Sapag, N \& Sapag, R. (Noviembre de 1991). Preparación y evaluación de proyectos (2 ed.). Mc Graw Hill. http://www.utntyh.com/wp-content/uploads/2013/03/Preparacion-YEvaluacion-De-Proyectos-Sapag-Sapag.pdf

Sautet, F. (2013). Local and systemic entrepreneurship: Solving the puzzle of entrepreneurship and economic development. Entrepreneurship Theory and Practice, 37(2): 387-402. https://journals.sagepub.com/doi/full/10.1111/j.1540-

6520.2011.00469.x\#articleCitationDownloadContainer

Shumpeter, J. (1934). The Theory of Economic Development. Journal of Comparative Research in Antropoloy and Sociology, 3(2), 137-148.

Thai, M. \& Turkina, E. (2014). Macro-level determinants of formal entrepreneurship vesus informal entrepreneurship. Journal of Business Venturing, 29(4): 490-510. https://ideas.repec.org/a/eee/jbvent/v29y2014i4p490-510.html

Timmons, J., Zacharakis, A. \& Spinelli, S. (2004). Business plans that works (2 ed). Mc Graw Hill.

Toro J. L. \& Ortegón A. A. (1999). Corriendo el telón del concepto emprendedor. Escuela de Administración de Negocios, (37), 134-142. https://journal.ean.edu.co/index.php/Revista/article/view/910/878

Praag, M. \& Versloot, P. H. (2007) What Is the Value of Entrepreneurship? A Review of Recent Research. IZA Discussion Papers (3014). https://ideas.repec.org/p/iza/izadps/dp3014.html2019b). 